\title{
Adsorption of diatomic halogen molecules on graphene: A van der Waals density functional study
}

\author{
A. N. Rudenko, , , 田 F. J. Keil, ${ }^{1}$ M. I. Katsnelson, ${ }^{2}$ and A. I. Lichtenstein ${ }^{3}$ \\ ${ }^{1}$ Institute of Chemical Reaction Engineering, Hamburg University \\ of Technology, Eissendorfer Str. 38, 21073 Hamburg, Germany \\ ${ }^{2}$ Radboud University Nijmegen, Institute for Molecules and Materials, \\ Heijendaalseweg 135, 6525 AJ Nijmegen, The Netherlands \\ ${ }^{3}$ Institute of Theoretical Physics, University of Hamburg, Jungiusstrasse 9, 20355 Hamburg, Germany
}

(Dated: October 24, 2018)

\begin{abstract}
The adsorption of fluorine, chlorine, bromine, and iodine diatomic molecules on graphene has been investigated using density functional theory with taking into account nonlocal correlation effects by means of vdW-DF approach. It is shown that the van der Waals interaction plays a crucial role in the formation of chemical bonding between graphene and halogen molecules, and is therefore important for a proper description of adsorption in this system. In-plane orientation of the molecules has been found to be more stable than the orientation perpendicular to the graphene layer. In the cases of $\mathrm{F}_{2}$, $\mathrm{Br}_{2}$ and $\mathrm{I}_{2}$ we also found an ionic contribution to the binding energy, slowly vanishing with distance. Analysis of the electronic structure shows that ionic interaction arises due to the charge transfer from graphene to the molecules. Furthermore, we found that the increase of impurity concentration leads to the conduction band formation in graphene due to interaction between halogen molecules. In addition, graphite intercalation by halogen molecules has been investigated. In the presence of halogen molecules the binding between graphite layers becomes significantly weaker, which is in accordance with the results of recent experiments on sonochemical exfoliation of intercalated graphite.
\end{abstract}

PACS numbers: 73.22.Pr, 73.20.Hb

\section{INTRODUCTION}

A monolayer of graphite, commonly known as graphene, the first truly two-dimensional crystal (oneatom-thick), which became experimentally available in the last years $\stackrel{1}{=}$ Today, graphene is at the focus of many research activities worldwide. Remarkable electronic properties of graphene make this material a promising candidate for a large variety of electronic applications $\underline{2.3}$

There have been a number of publications focused on theoretical investigation of covalent-, ionic- and metalbonded impurities on graphene (for example, see Refs.47). These studies are primarily based on the density functional theory (DFT) combined with standard functionals for electronic exchange-correlation effects, such as local density approximation (LDA) and generalized gradient approximation (GGA). The employment of (semi-)local approximations looks quite reasonable so far as the systems with either covalent or metallic bonding are concerned. However, in the case of weakly bounded systems, such as molecules or molecular compounds, dispersion forces play a significant role. Such interactions are essentially nonlocal and therefore cannot be properly taken into account using local or semi-local density functionals.

In this work, we will focus on molecular impurities on graphene. In particular, we consider diatomic halogen molecules on an ideal graphene lattice. The motivation for this choice is the following. First, these molecules themselves are covalently bonded and represent an example of realistic weakly reactive adsorbates.
Second, because of the relative chemical inertness of the molecules, the interaction between these molecules and the graphene sheet is rather weak and cannot be properly described within the electronic structure methods widely used nowadays in condensed matter physics (such as conventional LDA or GGA approximations in the density functional theories). Accurate calculations of the adsorption energies, which are necessary to judge how the impurities can be removed from the surface, are beyond these standard approaches which make their calculation a challenging problem. Last but not least, among the fundamental aspects of studying impurities on graphene, in some cases molecularly doped graphene represents itself an unavoidable outcome of its production processes. Recently Windekvist et $a l$. $\underline{\underline{8}}$ have reported a method of graphene fabrication based on sonochemical exfoliation of bromine-intercalated graphite. It was shown that ultrasonic treatment of graphite with absorbed bromine molecules results in intensive graphene-flakes formation. It is worth mentioning that intercalated graphite by itself has many potential applications $\underline{\underline{9}}$

In this paper we show that the adsorption of halogens on graphene is determined generally by the van der Waals (vdW) interactions. Besides the vdW interaction we also found a considerable ionic contribution for $\mathrm{F}_{2}$ and $\mathrm{I}_{2}$ adsorbates. We demonstrate that the ionic component of the interaction arises due to the electronic transfer from graphene to the molecules. Furthermore, the ionic interaction gives rise to a long-range tail of adsorption curves, which determines the adsorption at large distances.

In this work we also apply a van der Waals density 
functional approach (vdW-DF) $\underline{10,11}$ to investigate structural and energetic properties of graphite intercalated by different diatomic halogen molecules. We show that the interlayer distance and binding energies in graphite are also dependent on the exchange-correlation functional. Using vdW-DF method we have calculated interplanar binding of graphite layers in the presence of halogen molecules, which was found to be significantly weaker than the binding in pure graphite.

The rest of the paper is organized as follows. In Sec. II we briefly describe methods of the calculations. In Sec. III we present the results of adsorption of $\mathrm{F}_{2}, \mathrm{Cl}_{2}, \mathrm{Br}_{2}$, and $\mathrm{I}_{2}$ on graphene. Section IV is devoted to the analysis of electronic structure and ionic interaction between graphene and its adsorbates. The results of graphite intercalation by halogen molecules and corresponding discussion are given in Sec. V. In Sec. VI we briefly summarize our results.

\section{COMPUTATIONAL DETAILS}

\section{A. Crystal structure}

The structure of the systems under consideration was modelled using a supercell approach. The supercell consists of one graphene layer with a molecule on the top and a vacuum region to avoid spurious interaction between periodic images of the supercell in $z$-direction. In order to examine the effects of impurity concentration two supercells containing 24 and 32 carbon atoms per single molecule were considered. The corresponding graphene layers were constructed from rectangular $(3 \times 2 \sqrt{3}) a$ and $(4 \times 2 \sqrt{3}) a$ unit cells, respectively. The height of the supercells perpendicular to the surface was chosen to be 40 $\AA$. Investigating the supercells of such a size allows us to judge how the interactions between molecules can affect the system properties.

We used the lattice constant of graphene equal to $a=2.459 \AA$ in accordance with experimentally obtained value for graphite at low temperatures $\underline{12}$ The distortion of graphene layer in the presence of adsorbates was not taken into account. Since the interaction between the molecules and graphene is pretty weak the distortions of carbon lattice is supposed to be small, anyway. Interatomic distances in the halogen molecules were also taken from the experimental data $\underline{\underline{13}}$

Both in-plane and perpendicular-to-plane orientations of the molecules on graphene have been investigated. We considered five in-plane and three perpendicular-to-plane configurations corresponding to high-symmetry adsorption sites as illustrated in Fig [1.

\section{B. DFT computational details}

Ground-state energies and electronic density distributions have been calculated using the plane-wave pseu-

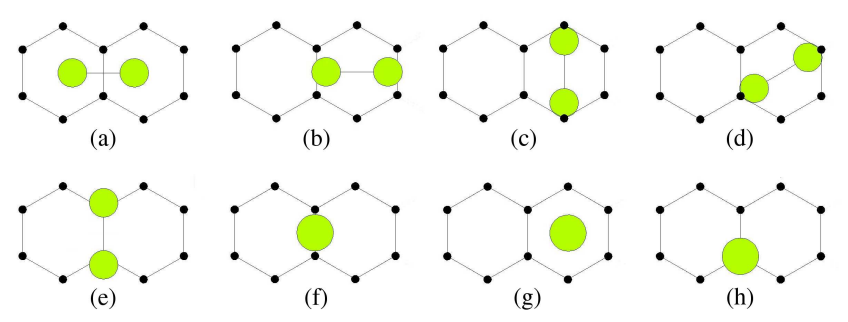

FIG. 1: Schematic representation of different high-symmetry adsorption sites for diatomic molecules on graphene surface (top view). In the case of in-plane orientation of the molecules the center of mass is located above: (a) a bridge site (the middle of C-C bond) along $x$-axis, (b) a hollow site along $x$-axis, (c) a hollow site along $y$-axis, (d) a hollow site along $\frac{\sqrt{3}}{2} x$-axis, (e) a bridge site along $y$-axis. Molecules perpendicular to the surface are located above: (f) a bridge site, (g) a hollow site, (h) a top site.

dopotential method as implemented in the QUANTUM-

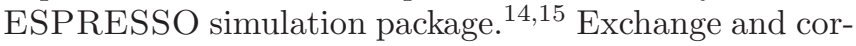
relation effects have been generally taken into account using vdW-DF nonlocal functional (see the next subsection for details). In order to obtain some comparative results the GGA functional in Pedrew-Burke-Ernzerhof (PBE) parametrization $\underline{16}$ also was used.

In our calculations we employed an energy cutoff of 30 Ry for the plane-wave basis and $300 \mathrm{Ry}$ for the charge density. Self-consistent calculations of the Kohn-Sham equations were carried out employing the convergence criterion of $10^{-8} \mathrm{Ry}$. For accurate Brillouin-zone integration the tetrahedron scheme $\frac{17}{\underline{17}}$ and $(16 \times 16 \times 1)$ Monkhorst-Pack k-point mesh 18 were used.

Geometry optimization algorithms were not used in our study. Atoms in graphene layer and interatomic distances in the molecules were assumed to be fixed. In order to find equilibrium distances of adsorbates we performed a set of explicit energy calculations for different separations from the surface. The most stable positions of the molecules were determined comparing the energies of different high-symmetry adsorption sites.

\section{Non-local corrections}

In the recent years several reasonable methods have been developed to improve the performance of DFT in description of sparse matter $\underline{19}-\underline{21}$ Most of them are based on the adiabatic connection fluctuation-dissipation framework (ACFD), which is a computationally very demanding approach and, in practice, is limited to small systems only $\stackrel{22,23}{2}$

Here, to calculate adsorption energies, we use an approach proposed by Dion et al $\stackrel{\underline{10}}{\underline{n}}$ The main idea of the method is that the ACFD correlation energy can be significantly simplified by applying a series of reasonable approximations resulting in the expression dependent on the electronic density only. Although being developed 
only recently, up to now this method has been widely verified on a large variety of examples and shows the transferability across a broad spectrum of interactions, such as ionic, covalent, and $\mathrm{vdW}, \underline{24}$

In the vdW-DF method, the exchange-correlation energy functional consists of several contributions,

$$
E_{x c}[n]=E_{x}^{r e v P B E}[n]+E_{c}^{L D A}[n]+E_{c}^{n l}[n],
$$

where the first term corresponds to the exchange part

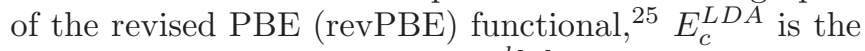
LDA correlation energy, and $E_{c}^{n l}[n]$ is the nonlocal correlation correction, which is calculated in the following way:

$$
E_{c}^{n l}=\frac{1}{2} \int d^{3} r d^{3} r^{\prime} n(r) \phi\left(r, r^{\prime}\right) n\left(r^{\prime}\right),
$$

where $n(r)$ is the electronic density and $\phi\left(r, r^{\prime}\right)$ is a function incorporating many-body density response (for details see Ref.10). It should be emphasized that in our work we evaluate the nonlocal correction $(\mathrm{Eq} 2)$ in a perturbative way, i.e. using only GGA-based (semi-local) electronic density distribution. This choice looks quite reasonable since it has been previously shown that the effects due to the lack of self-consistency are negligible. $\underline{11}$

\section{ADSORPTION OF MOLECULES ON GRAPHENE}

As we have already mentioned, we consider two different sizes of the supercell in order to figure out the influence of intermolecular binding on the investigated properties. We found that the adsorption energy at higher concentrations of halogens is slightly lower, however, this difference does not exceed $10 \mathrm{meV}$ and could be considered as insignificant. Due to this fact we do not expect any considerable changes in adsorption for different molecular concentrations. In the rest of this section we present the results only for the smaller supercell containing 24 carbon atoms.

We found the in-plane bridge position (Fig, 17) to be the most stable configuration for all the molecules under consideration. The binding energies for this particular geometry are shown in Fig 2 as a function of the distance between the surface and the center of mass of the molecule.

It is clear from Fig 2 that the account of the vdW interaction gives a considerable contribution to the binding energy for each molecule examined. One can see that the larger the radii of atoms in the molecule the stronger is the vdW binding to the surface. This fact can be easily figured out using the interpretation of the vdW interaction as an interaction of induced dipoles.

One can see from Fig 2 that the binding energy of the molecules decreases quite slowly with the distance. This fact is the most evident for the fluorine molecule (Fig 21), due to the relatively small energy scale. Moreover, the behavior of fluorine binding with graphene at large distances is fairly close to inverse proportionality and, therefore, indicates the presence of a strong ionic interaction. Indeed, at the distance of several angstroms the electronic density of graphene does not overlap with that of adsorbed molecules, which means that covalent interaction cannot occur in this case. Furthermore, the slowly vanishing tail of adsorption curves remains, even without taking into account the $\mathrm{vdW}$ correction, which confirms the ionic nature of such long-range interaction.

In the case of originally neutral constituents of the system, the ionic interaction could emerge only due to the charge transfer between them. Comparison of the binding energy for different halogens at relatively large distances (more than $6 \AA$ ) shows that in the absence of the vdW interaction the binding of graphene with fluorine is stronger than with other molecules. Assuming that this interaction is essentially ionic, we expect the larger charge transfer in $\mathrm{F}_{2}$ case. By the same reasoning the charge imbalance for $\mathrm{I}_{2}$ should be larger than for both $\mathrm{Br}_{2}$ and $\mathrm{Cl}_{2}$. A more detailed discussion on the ionic interaction will be given in the next section.

For comparison we also show in Fig 2 the results obtained by the standard semi-local GGA functional in PBE parametrization. While the equilibrium distances of vdW-DF and PBE approaches agree reasonably well, the adsorption energies in the case of PBE are highly overestimated. The latter fact is not surprising since GGA-like functionals do not incorporate nonlocal correlation effects, which are responsible for the vdW interaction. It should be also pointed out that PBE functional yields the wrong asymptote for the adsorption curves with the exception of fluorine, where the vdW contribution at the large distances is not as large as the ionic one.

Adsorption energies for different orientations of the molecules are summarized in Table [ For both in-plane and perpendicular-to-plane orientations the less stable configurations correspond to the hollow adsorption site (Fig 1b,c,d,g). This result is quite reasonable as long as the $\mathrm{vdW}$ interaction dominates in the system. Indeed, at the same distance the strength of the $\mathrm{vdW}$ interaction is determined by electronic densities corresponding to interacting components of the system. $\underline{\underline{19}}$ It turns out that the hollow site corresponds to the minimum of electronic density in graphene, which accounts for the lower vdW interaction in this case.

The difference between various adsorption energies of in-plane (or perpendicular-to-plane) orientations of the molecules is small. This is especially clear for the rotated molecules at the hollow adsorption site (Fig \b,c,d). In contrast, the adsorption energies of in-plane orientations are sufficiently different from the energies of perpendicular-to-plane orientations, exceeding thermal energy at room temperature $(\sim 25 \mathrm{meV})$.

We assume that the molecular bond length cannot be significantly changed during the adsorption process because the obtained molecule-surface interaction is much weaker than the intramolecular binding in halogens $\underline{13}$ Due to the same reason we do not expect the dissocia- 


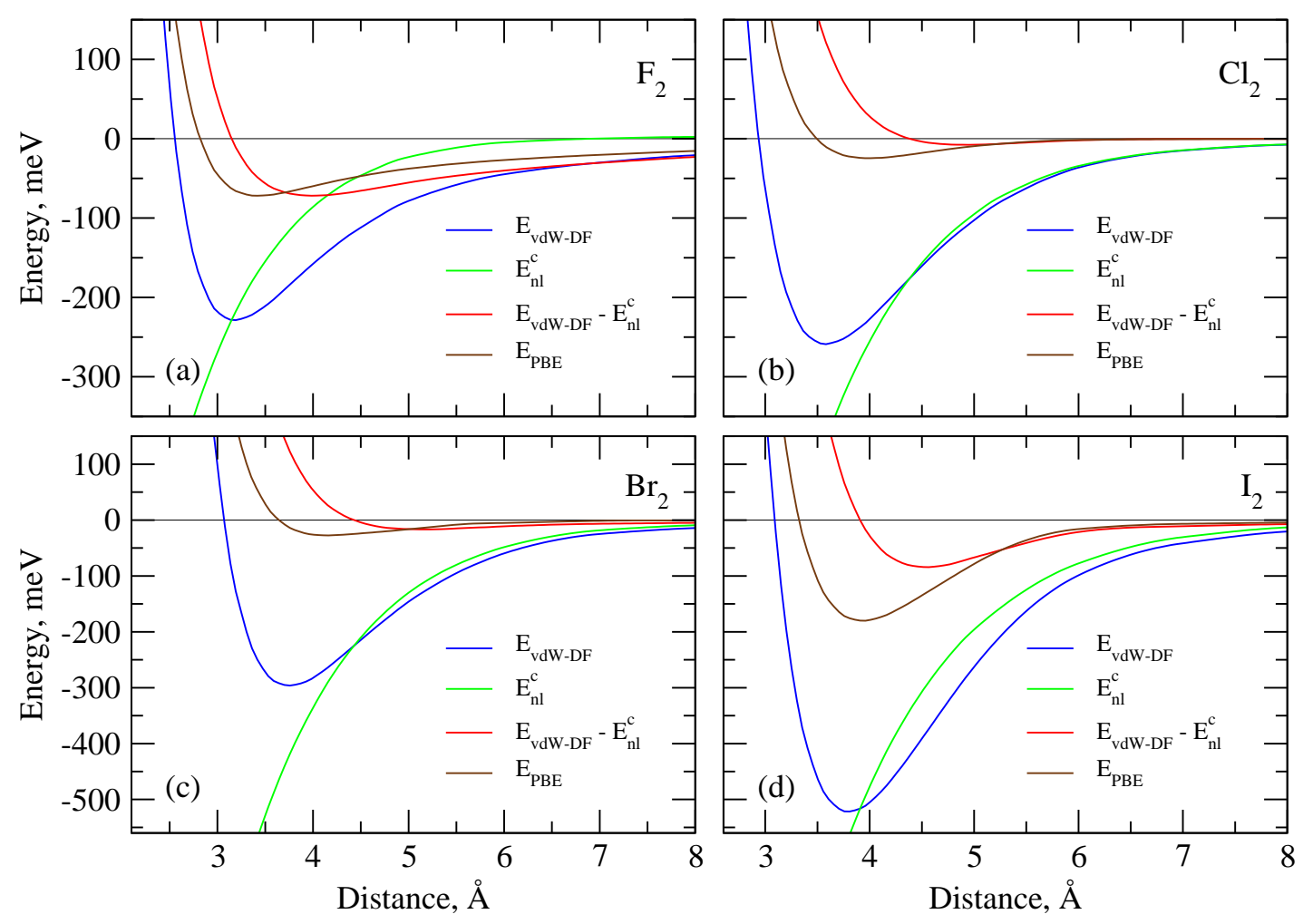

FIG. 2: Adsorption energies of halogen molecules on graphene as a function of the separation between graphene and the center of mass of the molecules: (a) $\mathrm{F}_{2}$, (b) $\mathrm{Cl}_{2}$, (c) $\mathrm{Br}_{2}$, (d) $\mathrm{I}_{2}$. Blue, green and red curves correspond to the total binding energy calculated within vdW-DF approach, nonlocal vdW correction (EqL2), and to the binding energies excluding vdW correction, respectively. Brown curve corresponds to the PBE binding energy. Adsorption curves are shown only for the most stable molecular configuration (Fig[1).

TABLE I: Binding energies in graphene-halogen system for different high-symmetry adsorption sites of the molecules, and corresponding equilibrium distances (in parentheses). Adsorption sites denoted as in Fig 1 Values are given in meV and $\AA$, respectively.

\begin{tabular}{ccccccccc}
\hline \hline & \multicolumn{3}{c}{ in-plane } & \multicolumn{3}{c}{ perpendicular-to-plane } \\
\cline { 2 - 5 } \cline { 7 - 9 } & $\mathrm{a}$ & $\mathrm{b}$ & $\mathrm{c}$ & $\mathrm{d}$ & $\mathrm{e}$ & $\mathrm{f}$ & $\mathrm{g}$ & $\mathrm{h}$ \\
\hline $\mathrm{F}_{2}$ & -231 & -227 & -225 & -226 & -222 & -214 & -187 & -218 \\
& $(3.17)$ & $(3.23)$ & $(3.24)$ & $(3.23)$ & $(3.26)$ & $(3.47)$ & $(3.70)$ & $(3.46)$ \\
$\mathrm{Cl}_{2}$ & -259 & -248 & -249 & -250 & -251 & -208 & -193 & -210 \\
& $(3.58)$ & $(3.65)$ & $(3.64)$ & $(3.65)$ & $(3.63)$ & $(4.21)$ & $(4.34)$ & $(4.21)$ \\
$\mathrm{Br}_{2}$ & -296 & -289 & -287 & -285 & -288 & -229 & -207 & -214 \\
& $(3.74)$ & $(3.77)$ & $(3.77)$ & $(3.77)$ & $(3.78)$ & $(4.51)$ & $(4.67)$ & $(4.45)$ \\
$\mathrm{I}_{2}$ & -523 & -514 & -508 & -501 & -512 & -375 & -352 & -379 \\
& $(3.80)$ & $(3.84)$ & $(3.83)$ & $(3.83)$ & $(3.81)$ & $(4.74)$ & $(4.85)$ & $(4.73)$ \\
\hline \hline
\end{tabular}

tive adsorption of halogens on graphene, which usually occurs for light molecules on metallic surfaces, 26 We note, however, that a careful description of this phenomenon requires atomic dynamics to be taken into account, and therefore should be investigated separately.

\section{ELECTRONIC STRUCTURE AND IONIC INTERACTION}

In the previous section we have shown that the vdW interaction gives a significant contribution to the adsorption energy, and, therefore, plays an important role in formation of bonding between halogen molecules and graphene. However, besides the vdW part there is another source of interaction which gives rise to the bonding. In particular, this contribution especially important for fluorine and iodine adsorbates. In order to clarify the 


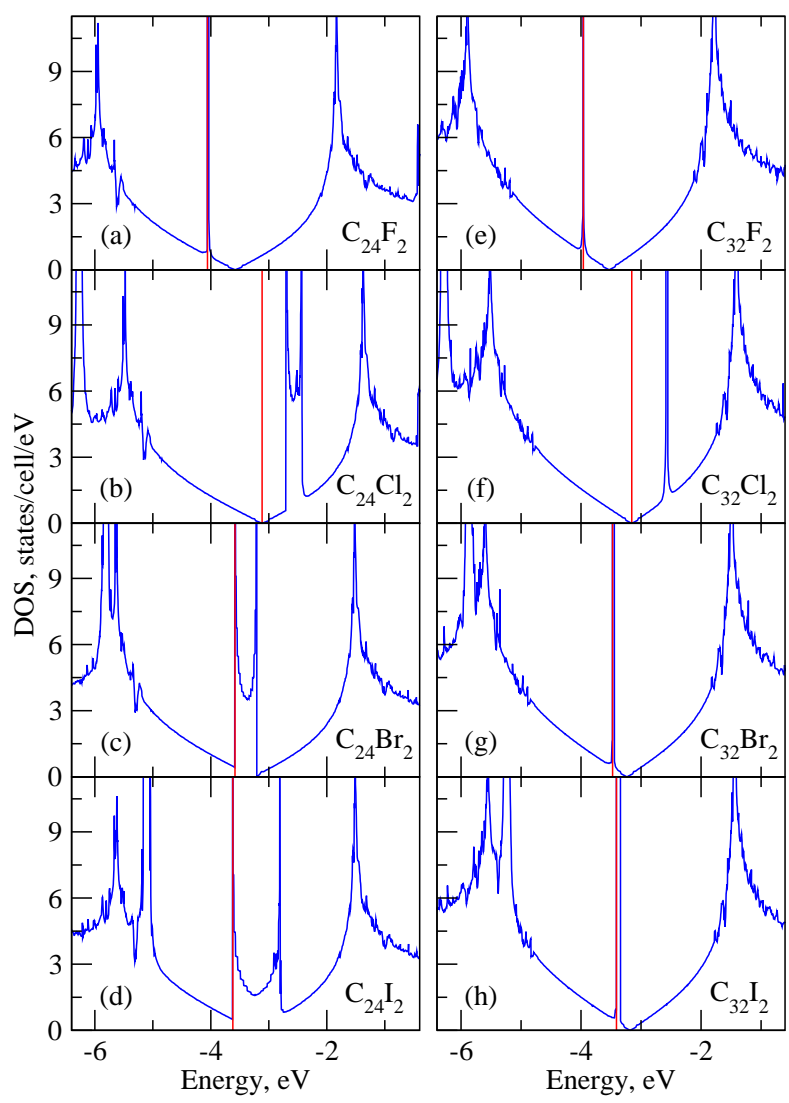

FIG. 3: Total electronic density of states of halogen molecules adsorbed on graphene calculated for different supercells. Left (right) panel corresponds to the supercell containing 24 (32) carbon atoms. Vertical line accentuates the Fermi energy. Densities of states are shown for the respective ground state distances obtained without vdW corrections (see Fig[2).

nature of such an interaction let us analyze the density of electronic states in the absence of the vdW forces.

In Fig 3 we have plotted the density of states (DOS) for each considered molecule in two different supercells, which is calculated for the lowest-energy structural state without taking into account nonlocal correction to correlation energy (excluding third term in Eq1). In the vicinity of the Fermi level DOS exhibits resonances which are entirely localized on $p$-orbitals of halogen molecules. The main differences between the spectra for different molecular concentrations are related to the width of impurity bands. In both cases the impurity states close to the Fermi level remain unoccupied.

Analysis of the electronic bands shows that the impurity states close to the Fermi level correspond only to one electron per spin, i.e. to one electronic level in non spin-polarized case. This means that the observed impurity band at high concentrations of molecules cannot be associated with the hybridization between molecular and graphene orbitals. In fact, broadening of the impurity level caused by the hybridization between molecular $p_{x}$-orbitals from different images of the supercell forming parabolic-like DOS, which is very similar to that of a one-dimensional chain. Indeed, for the most energetically favorable configuration (Fig $1 \mathrm{a}$ ) the distance between molecules in $x$-direction of the supercell is smaller than the distance in other directions. As the distance between atoms in the molecule increases, the separation between different molecules becomes smaller, which leads to hybridization of molecular orbitals and broadening of the electronic levels. This also accounts for the absence of broad impurity bands for lower molecular concentrations where the lateral interactions between molecules are negligible. The shape of the band for the smallest supercell (24 carbon atoms) clearly shows formation of onedimensional band, with typical divergencies (van Hove singularities) at the edges. The width of this band, that is, the distance between the singularities, is estimated as twice the effective hopping parameter.

For low concentrations, the impurity states are located well below or above the conical (Dirac) point, which is easily recognizable in the density of states. However, this is not the case for $\mathrm{Br}_{2}$ and $\mathrm{I}_{2}$ adsorbed at their highest concentrations (Fig $3 \mathrm{r}, \mathrm{d})$. In this situation, the Dirac point lies inside the impurity band. Moreover, since the impurity states for $\mathrm{Br}_{2}$ and $\mathrm{I}_{2}$ are broadened enough, they can be considered as conduction bands leading to a metallic behavior of the system. Therefore, the conductivity of graphene can be adjusted varying the concentration of $\mathrm{Br}_{2}$ and $\mathrm{I}_{2}$ adsorbates. There are preliminary experimental evidences that doping by iodine can essentially increase the conductivity of graphene $e^{27}$

As can be seen from Fig 3 the Fermi level is shifted below the Dirac point for the all molecules except chlorine. A shift downward means that the electrons are donated by graphene to $\mathrm{F}_{2}, \mathrm{Br}_{2}$ and $\mathrm{I}_{2}$ molecules (acceptors). As discussed above, the electronic transfer is not surprising for the investigated systems. It turns out that the assumption of an ionic interaction between graphene and its adsorbates, given in the previous section, is in a good agreement with the peculiarities of electronic structure.

For a more detailed description of the ionic interaction, let us analyze the charge transfer phenomenon. As the molecules come closer to graphene, redistribution of the electronic density results in the formation of an interface dipole layer, which is responsible for the ionic interaction in the system. In Fig 4 we show the plane-averaged density differences $\Delta n(z)$ calculated along $z$-axis of the supercell for each investigating molecule at the distance of $5 \AA$ from the surface. The most noticeable feature of Fig 4 is that there are almost no changes in electron density for chlorine in comparison with other molecules. This seems to be a reasonable result because the Fermi energy coincides in this case with the Dirac point. The electron redistribution $\Delta n(z)$ for the other molecules has nearly the same shape, which is fairly close to the shape of a typical dipolar distribution. $\Delta n(z)$ exhibits a main minimum (maximum) corresponding to localization of the charge near the carbon layer (molecule). In the in- 


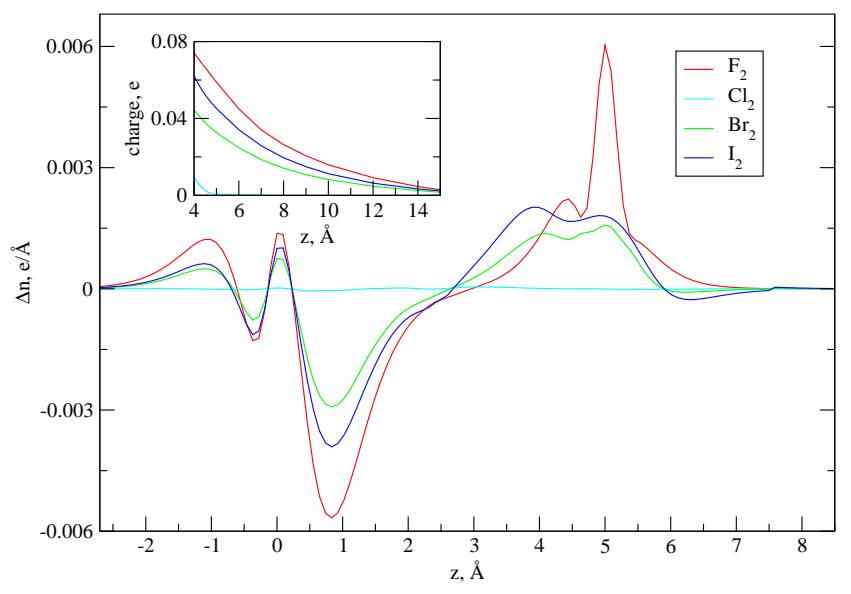

FIG. 4: In-plane-averaged electron density difference calculated along $z$-axis of the supercell for $\mathrm{F}_{2}, \mathrm{Cl}_{2}, \mathrm{Br}_{2}$, and $\mathrm{I}_{2}$ molecules on graphene. $z=0$ corresponds to position of the carbon layer. In the inset an integrated amount of the charge transferred from the surface to the molecules as a function of the distance is displayed.

set of Fig 4 we also show an integrated charge, transferred from graphene to the molecules as a function of the distance between them $\stackrel{28}{2}$ As expected, the amount of the transferred charge decreases as the distance between graphene and the molecules increases. As can be seen from Fig, at the equilibrium distance fluorine molecule accepts more electrons than either $\mathrm{I}_{2}$ or $\mathrm{Br}_{2}$. This fact completely agrees with our previous results and explains the large ionic interaction between fluorine molecule and graphene.

\section{INTERCALATION OF GRAPHITE}

It is known that the van der Waals interaction plays an important role in understanding and proper description of the interplanar bonding in graphite. ${ }^{29-31}$ In spite of the fact that the standard local density approximation (LDA) completely misses dispersion interaction (at least for long distances), it reproduces correctly the interlayer spacing in graphite and even the binding energy in some cases $\frac{30}{3}$ This surprising result seems, however, to be accidental because it is impossible to reproduce accurately the experimental compressibility of graphitic structures within the LDA 32 Recently it was shown 33 that the vdW-DF approach yields not only the proper distance between graphite sheets, but also the binding energy, which is very close to the experimental estimations available today ${ }^{34,35}$ The vdW-DF method was also successfully applied to the investigation of energetics in potassium-intercalated graphite yielding a good agreement with experiment. 36

In this section we use the vdW-DF approach to describe structural and energetic properties of graphite in-
TABLE II: Equilibrium interlayer distances, absorption energies, and interlayer binding energies for the pure and halogenintercalated graphite. Absorption and binding energies are given in meV per absorbed molecule and in meV per carbon atoms in the supercell, respectively.

\begin{tabular}{cccccc}
\hline \hline & $\mathrm{C}_{24}$ & $\mathrm{C}_{24} \mathrm{~F}_{2}$ & $\mathrm{C}_{24} \mathrm{Cl}_{2}$ & $\mathrm{C}_{24} \mathrm{Br}_{2}$ & $\mathrm{C}_{24} \mathrm{I}_{2}$ \\
\hline$d, \AA$ & 3.6 & 6.0 & 7.0 & 7.5 & 7.4 \\
$\Delta E_{\text {abs }}, \mathrm{meV} / \mathrm{mol}$ & 0 & 648 & 605 & 542 & 114 \\
$\Delta E_{\text {bind }}, \mathrm{meV} / \mathrm{C}$ & -49.5 & -12.9 & -13.6 & -14.6 & -22.9 \\
\hline \hline
\end{tabular}

tercalated by halogen molecules. We consider graphite in $A B$-stacking containing 48 carbon atoms and 2 halogen molecules per unit cell. Absorbed molecules are assumed to be uniformly distributed over graphite occupying inplane bridge sites in accordance with the most stable position on graphene layer (Fig प $\mathrm{h}$ ). Other parameters of the calculations were taken to be the same as described in Sec.IIB.

It was recently demonstrated that graphene sheets are impermeable even to light gases including helium: $\underline{37,38}$ For this reason, we cannot expect that the intercalation of halogen molecules in graphite is a result of a penetration of the molecules underneath the top surface layer. On the contrary, we suppose that the formation of intercalated graphite involves a consecutive exfoliation of the whole graphite structure.

In Table II we summarize equilibrium distances, absorption energies, and interlayer binding energies calculated for the investigated systems $\underline{39}$ The interlayer distance in intercalated graphite is approximately twice larger than in the pure case. We note, however, that the estimation of the interlayer spacing is not very accurate since we ignore in our calculations a distortion of the intercalated graphite lattice, which will inevitably take place in the real situation. The absorption energies given in Table I are positive because their calculation involves a positive energy of the exfoliation process. It should be pointed out that among the other considered molecules, iodine molecule has a minimal absorption energy value.

The binding energies obtained for the pure graphite are in a good agreement with the previous vdW-DF calculations $: \frac{33,36}{3}$ Comparing the interlayer binding for the pure and for the intercalated graphite, we conclude that the binding becomes significantly weaker in the presence of halogen molecules. Moreover, the binding energy is lower for the more massive molecules because of their stronger interaction with the carbon layers, as discussed in Sec.III. Our results are in a qualitative agreement with the previous LDA calculations for bromine-intercalated graphite $\stackrel{8}{*}$ Although the interlayer binding for our particular impurity concentration seems to be overestimated (in absolute values) in comparison with the LDA results, the general tendency is reproduced quite well and allows to confirm the interpretation of the experiment on sonochemical exfoliation of intercalated graphite given in Ref:8. 


\section{CONCLUSION}

We have performed a first-principles investigation of the adsorption of diatomic halogen molecules on graphene. We have shown that a major part of the binding energy in the system corresponds to van der Waals interaction, and, therefore, cannot be properly described by means of standard (semi-)local approximations to exchange-correlation energy. It has been found that even at the large distances (up to $10 \AA$ ) there is a non-zero interaction between graphene and $\mathrm{F}_{2}, \mathrm{Br}_{2}$, $\mathrm{I}_{2}$ molecules. This interactions are of ionic nature and arise due to the charge transfer from graphene to the molecules.

In contrast to $\mathrm{F}_{2}$ and $\mathrm{Cl}_{2}$ adsorbates, the densities of states for graphene in the presence of $\mathrm{Br}_{2}$ and $\mathrm{I}_{2}$ molecules exhibit impurity bands right above the Fermi level, which can result, for large enough concentration, in the metallization of graphene. Moreover, the width of the impurity band is directly dependent on the impurity concentration.

Finally, investigating properties of intercalated graphite we have found that interlayer binding becomes significantly weaker than in pure graphite. This is in agreement with the previous experimental and theoretical investigations of intercalated graphite.

\section{ACKNOWLEDGMENTS}

We would like to thank Irina Grigorieva and Tim Wehling for helpful discussions. We also appreciate the assistance of Claudia Ambrosch-Draxl and Dmitrii Nabok for providing a very efficient algorithm for the calculation of vdW-DF correlation energies by MonteCarlo method. The authors acknowledge support from the Cluster of Excellence "Nanospintronics" (Hamburg, Germany) and from Stichting voor Fundamenteel Onderzoek der Materie (FOM), the Netherlands.
* rudenko@tu-harburg.de

1 K. S. Novoselov, A. K. Geim, S. V. Morozov, D. Jiang, Y. Zhang, S. V. Dubonos, I. V. Grigorieva, and A. A. Firsov, Science 306, 666 (2004).

2 A. K. Geim and K. S. Novoselov, Nature Materials 6, 183 (2007).

3 A. H. Castro Neto, F. Guinea, N. M. R. Peres, K. S. Novoselov, and A. K. Geim, Rev. Mod. Phys. 81, 109 (2009).

4 T. O. Wehling, M. I. Katsnelson, and A. I. Lichtenstein, Phys. Rev. B. 80, 085428 (2009).

5 T. O. Wehling, A. I. Lichtenstein, and M. I. Katsnelson, Appl. Phys. Lett. 93, 202110 (2009).

${ }^{6}$ P. A. Khomyakov, G. Giovannetti, P. C. Rusu, G. Brocks, J. van den Brink, and P. J. Kelly, Phys. Rev. B. 79, 195425 (2009).

7 H. Johll, H. C. Kang, and E. S. Tok, Phys. Rev. B. 79, 245416 (2009).

8 E. Widenkvist, D. W. Boukhvalov, S. Rubino, S. Akhtar, J. Lu, R. A. Quinlan, M. I. Katsnelson, K. Leifer, H. Grennberg, and U. Jansson, J. Phys. D: Appl. Phys. 42, 112003 (2009).

9 T. Enoki, M. Suzuki, M. Endo, Graphite intercalation compounds and applications, (Oxford University Press, Oxford and New York, 2003).

10 M. Dion, H. Rydberg, E. Schröder, D. C. Langreth, and B. I. Lundqvist, Phys. Rev. Lett. 92, 246401 (2004).

11 T. Thonhauser, V. R. Cooper, S. Li, A. Puzder, P. Hyldgaard, and D. C. Langreth. Phys. Rev. B 76, 125112 (2007).

12 Y. Baskin and L. Meyer, Phys. Rev. 100, 544 (1955).

13 L. Pauling, The nature of the chemical bond and the structure of molecules and crystals: an introduction to modern structural chemistry, 3rd edition (Ithaca, NY: Cornell University Press, 1960).

${ }^{14}$ P. Giannozzi, S. Baroni, N. Bonini, M. Calandra, R. Car, C. Cavazzoni, D. Ceresoli, G. L. Chiarotti, M. Cococ- cioni, I. Dabo, A. Dal Corso, S. de Gironcoli, S. Fabris, G. Fratesi, R. Gebauer, U. Gerstmann, C. Gougoussis, A. Kokalj, M. Lazzeri, L. Martin-Samos, N. Marzari, F. Mauri, R. Mazzarello, S. Paolini, A. Pasquarello, L. Paulatto, C. Sbraccia, S. Scandolo, G. Sclauzero, A.P. Seitsonen, A. Smogunov, P. Umari, and R. M. Wentzcovitch, J. Phys.: Condens. Matter, 21, 395502 (2009).

15 The pseudopotentials used in this work were taken from QuANTUM-ESPRESSO web page http://www.quantum-espresso.org

16 J. P. Perdew, K. Burke, and M. Ernzerhof, Phys. Rev. Lett. 77, 3865 (1996).

17 P. E. Blöchl, O. Jepsen, and O. K. Andersen, Phys. Rev. B. 49, 16223 (1994).

18 H. J. Monkhorst and J. D. Pack, Phys. Rev. B. 13, 5188 (1976).

19 Y. Andersson, D. C. Langreth, and B. I. Lundqvist, Phys. Rev. Lett. 76, 102 (1996).

20 P. L. Silvestrelli J. Phys. Chem. A 113, 5224 (2009).

${ }^{21}$ H.-V. Nguyen and S. de Gironcoli, Phys. Rev. B 79, 115105 (2009).

22 Y. M. Niquet, M. Fuchs, and X. Gonze, Phys. Rev. A 68, 032507 (2003).

23 J. Harl and G. Kresse, Phys. Rev. B 77, 045136 (2008).

24 D. C. Langreth, B. I. Lundqvist, S. D. Chakarova-Käck, V. R. Cooper, M. Dion, P. Hyldgaard, A. Kelkkannen, J. Kleis, Lingzhu Kong, Shen Li, P. G. Moses, E. Murray, A. Puzder, H. Rydberg, E. Schröder, and T. Thonhauser, J. Phys.: Condens. Matter 21, 084203 (2009).

25 Y. Zhang and W. Yang, Phys. Rev. Lett. 80, 890 (1998).

26 A. Gross, Theoretical surface science: a microscopic perspective (Springer-Verlag Berlin Heidelberg, 2003), p.171.

27 I. V. Grigorieva, private communication.

${ }^{28}$ We calculate the charge transfer following the procedure used in Ref 6. This procedure implies that the charge $q$ can be estimated by integrating plane-averaged density difference $\Delta n(z)$ as follows: $q=-|e| \int_{z_{0}}^{\infty} d z \Delta n(z)$, where $z_{0}$ 
corresponds to an interface point so that $\Delta n\left(z_{0}\right)=0$

29 J.-C. Charlier, X. Gonze, and J.-P. Michenaud, Europhys. Lett. 28, 403 (1994).

${ }^{30}$ L. A. Girifalco and M. Hodak, Phys. Rev. B 65, 125404 (2002).

31 Y. J. Dappe, M. A. Basanta, F. Flores, and J. Ortega, Phys. Rev. B 74, 205434 (2006).

32 A. H. R. Palser, Phys. Chem. Chem. Phys. 1, 4459 (1999).

33 S. D. Chakarova-Käck, E. Schröder, B. I. Lundqvist, and D. C. Langreth, Phys. Rev. Lett. 96, 146107 (2006).

34 L. X. Benedict, N. G. Chopra, M. L. Cohen, A. Zettl, S. G. Louie, and V. H. Crespi, Chem. Phys. Lett. 286, 490 (1998).

35 R. Zacharia, H. Ulbricht, and T. Hertel, Phys. Rev. B 69, 155406 (2004).

${ }^{36}$ E. Ziambaras, J. Kleis, E. Schröder, and P. Hyldgaard,
Phys. Rev. B 76, 155425 (2007).

37 J. S. Bunch, S. S. Verbridge, J. S. Alden, A. M. van der Zande, J. M. Parpia, H. G. Craighead, and P. L. McEuen, Nano Lett. 8, 2458 (2008).

38 O. Leenaerts, B. Partoens, and F. M. Peeters, Appl. Phys. Lett. 93, 193107 (2008).

39 We calculate the absorption energy of intercalated graphite as follows: $\Delta E_{a b s}=-\Delta E_{\text {form }}^{C_{48}}+\Delta E_{\text {form }}^{C_{48} X_{4}}$, where the first term corresponds to the energy of the pure graphite formation from a number of non-interacting graphene sheets, and the seconds term is the energy of the intercalated graphite formation. In turn, the binding energy of intercalated graphite we estimate as: $\Delta E_{\text {bind }}=\Delta E_{\text {form }}^{C_{48} X_{4}}-2 \Delta E_{\text {form }}^{C_{24} X_{2}}$, where second term is the binding energy in graphenehalogen system. $\mathrm{X}_{2}=\mathrm{F}_{2}, \mathrm{Cl}_{2}, \mathrm{Br}_{2}, \mathrm{I}_{2}$. 